\title{
Overview: Importance of Network Security and Future Issues
}

\author{
El Mutaz Mahdi Osman Elawad, Dr.Haala El Dawo, \\ Department of Communications, Faculty of Engineering, Al-Néelain University
}

\begin{abstract}
Network security has become more important to personal computer users, organizations, and the military. With the advent of the internet, security became a major concern and the history of security allows a better understanding of the emergence of security technology. The internet structure itself allowed for many security threats to occur. The architecture of the internet, when modified can reduce the possible attacks that can be sent a cross the network. Knowing the attack methods, allows for the appropriate security to emerge. Many businesses secure themselves from the internet by means of firewalls and encryption mechanisms. The businesses create an "intranet" to remain connected to the internet but secured from possible threats. The entire field of network security is vast and in an evolutionary stage. The range of study encompasses a brief history dating back to internet's beginnings and the current development in network security. In order to understand the research being performed today, background knowledge of the internet, its vulnerabilities, attack methods through the internet, and security technology is important and therefore they are reviewed.
\end{abstract}

\section{Introduction}

The world is becoming more interconnected with the advento the Internet and new networking technology. There is a large amount of personal, commercial, military, and government information on networking infrastructures world wide. Network security is be coming of great importance because of in tellectual property that can be easily acquired through the internet.

There are currently two fund amentally different networks, data networks and synchronous network comprised of switches. The internet is considered a data network. Since the current data network consists of computer-based routers, information can be obtained by special programs, such as "Trojan horses," planted in the routers. The synchronous network that consists of switches does not buffer data and therefore are not threatened by attackers. That is why security is emphasized in data networks, such as the internet, and other networks that link to the internet.

The vast topic of network security is analyzed by researching the following:

1. History of security in networks

2. Internet architecture and vulnerable security aspects of the Internet

3. Types of internet attacks and security methods

4. Security for networks with internet access

5. Current development in network security

Hardware and software

Based on this paper, the future of network security is forecasted. New trends that are emerging will also be considered to understand where network security s heading.

\section{Network Security}

System and network technology is a key technology for a wide variety of applications. Security is crucial to networks and applications. Although, network security is a critical requirement in emerging networks, there is a significant lack of security methods that can be easily implemented.

There exists a "communication gap" between the developers of security technology and developers of networks. Network design is a well-developed process that is based on the Open Systems Interface (OSI) model. The OSI model has several advantages when designing networks. It offers modularity, flexibility, ease-of-use, and standardization of protocols. The protocols of different layers can be easily combined to create stacks which allow modular development. The implementation of individual layers can be changed later without making other adjustments, allowing flexibility in development. In contrast to network design, secure network design is not a well- developed process. There is n't a methodology to manage the complexity of security requirements. Secure network design does not contain the same advantages as network design.

When considering network security, it must be emphasized that the whole network is secure. Network security does not only concern the security in the computers a teach end of the communication chain. When transmitting data the communication channel should not be vulnerable to attack. A possible hacker could target the communication channel, obtain the data, decrypt it and re-insert a false message. Securing the network is just as important as securing the computers and encrypting the message.

When developing a secure network, the following need to be considered[1]: 
1. Access-authorized users are provided the means to communicate to and from a particular network

2. Confidentiality-Information in the network remains private

3. Authentication-Ensure the users of the network are who they say they are

4. Integrity -Ensure the message has not been modified in transit

5. Non-repudiation-Ensure the user does not

refute that he used the network.

An effective network security plan is developed with the under standing of security issues, potential attackers, needed level of security, and factors that make a network vulnerable to attack [1]. The steps involved in understanding the composition of a secure network, internet or otherwise, is followed through out this research endeavor.

To lessen the vulnerability of the computer to the network there are many products available. These tools are encryption, authentication mechanisms, intrusion-detection, security management and firewalls. Businesses throughout the world are using a combination of some of the se tools. "Intranets" are both connected to the internet and reasonably protected from it. The internet architecture itself leads to vulner abilities in the network. Understanding the security issues of the Internet greatly assists in developing new security technologies and approaches for networks with Internet access and Internet security itself.

The types of attacks through the internet need to also be studied to be able to detect and guard against them. Intrusion detection systems are established based on the types of attacks most commonly used. Network intrusions consist of packets that are introduced to cause problems for the following reasons:

- To consume resources uselessly

- To interfere with any system resource's intended function

- To gain system knowledge that can be exploited in later attacks

The last reason for a network intrusion is most commonly guarded against and considered by most as the only intrusion motive. The other reasons mentioned need to bethwarted as well.

Typical security currently exists on the computers connected to the network. Security protocols sometime susually appear as part of a single layer of the OSI network reference model. Current work is being performed in using a layered approach to secure network design. The layers of the security model correspond to the OSI model layers. This security approach leads to an effective and efficient design which circumvents some of the common security problems.

\section{Differentiating Data Security and Network Security}

Data security is the aspect of security that allows a client's data to be transformed into unintelligible data for transmission. Even if this unintelligible data is intercepted, a key is needed to decode the message. This method of security is effective to a certain degree. Strong cryptography in the past can be easily broken today. Cryptographic methods have to continue to advanced auto the advancement of the hackers as well.

When transferring cipher text over a network, it is helpful to have a secure network. This will allow for the cipher text to be protected, so that it is less likely for many people to even attempt to break the code. A secure network will also prevent someone from inserting unauthorized messages into the network. Therefore, hard ciphers are need edas well as attack-hard networks[2].

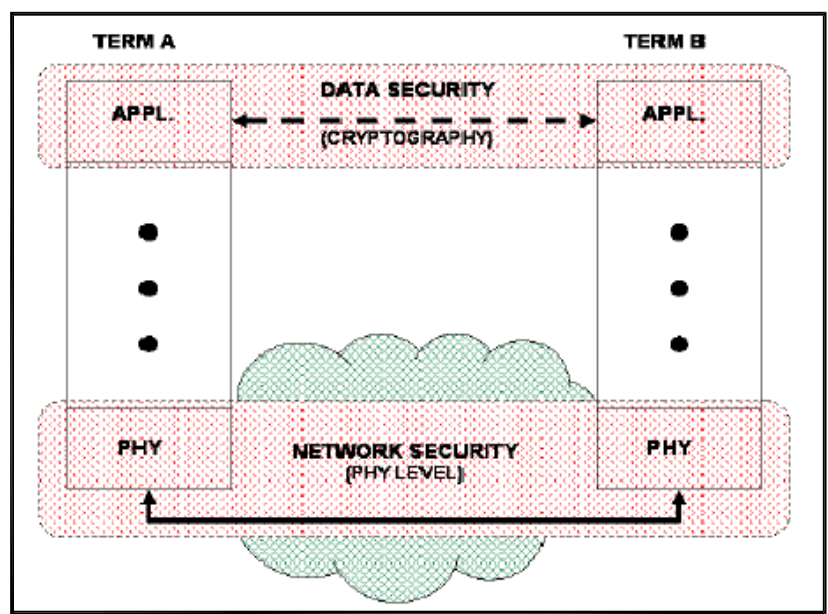

Figure 1: Based on the OSI model, data security and network security have a different security function [2].

There lation ship of network security and data security to the OSI model is shown in Figure1. 
It can be seen that the cryptography occurs at the application layer; therefore the application writers area ware of its existence. The user can possibly choose different methods of data security. Network security is mostly contained with in the physical layer. Layers above the physical layer are also used to accomplish the network security required [2]. Authentication is performed on a layer above the physical layer. Network security in the physical advance due to, attack detection mechanisms, and intelligent countermeasure strategies[2].

\section{History of network security}

Recent interest in security was fueled by the crime committed by Kevin Mitnick. Kevin Mitnick committed the largest computer-related crime in U.S. history [3]. The losses were eighty million dollars in U.S. intellectual property and source code from a variety of companies[3]. Since then, information security came into the spotlight.

Public networks are being relied upon to deliver financial and personal information. Due to the evolution of information that is made available through the internet, information security is also required to evolve. Due to Kev in Mitnick's of fense, companies are emphasizing security for the intellectual property. Internet has been a driving force for data security improvement.

Internet protocols in the past were not developed to secure themselves. Within the TCP/IP communications tack, security protocols are not implemented. This leaves the internet open to attacks. Modern developments in the internet architecture have made communication more secure.

\section{Brief History of Internet}

The birth of the interne takes place in 1969 when Advanced Research Projects Agency Network (ARPA Net) is commissioned by the department of defense (DOD) for research in networking. The ARPANET is a success from the very beginning. Although originally designed to allow scientists to share data and access remote computers,e-mail quickly becomes the most popular application. The ARPANET becomes a high-speed digital post office as people use it to collaborateonre search projects and discuss topics of various interests. The Inter Networking Working Group becomes the first of several standards-setting entities to govern the growing network [10]. Vinton Cerfi selected the first chairman of the INWG, and later becomes known as a" Father of the Internet." [10]

In the1980s, BobKahn and Vinton Cerf are key members of a team that create TCP/IP, the common language of all Internet computers. For the first time the loose collection of networks which made up the ARPANET is seen as an "Internet", and the Internet as we know it today is born. Themid-80smarksaboominthepersonal computer and super-minicomputer industries. The combination of inexpensive desktop machines and powerful, network-ready servers allows many companies to join the Internet for the first time. Corporations begin to use the Internet to communicate with each other and with their customers.

In the1990s, the internet began to become available to the public. The World Wide Web was born. Netscape and Microsoft were both competing on developing a browser for the internet. Internet continues to grow and surfing the internet has become equivalent to TV viewing for many users.

\section{Security Timeline}

Several key events contributed to the birth and evolution of computer and network security. The time line can be started as far back as the 1930s. Polish cryptographer screated an enigma machine in 1918 that converted plain messages to encrypted text. In 1930, Alan Turing, a brilliant mathematician broke the code for the Enigma. Securing communications was essential in World War II.

In the $1960 \mathrm{~s}$, the term "hacker" is coined by a couple of Massachusetts Institute of Technology (MIT) students. The Department of Defense began the ARPA Net, which gains popularity as a conduit for the electronic exchange of data and information[3]. This paves the way for the creation of the carrier network known today as the Internet. Duringthe1970s,theTelnet protocol was developed. This opened the door for publicuse of data networks that were originally restricted to government contractors and academic researchers [3].

During the1980s, the hackers and crimes relating to computers were beginning to emerge. The 414 gang are raided by authorities after a nine-day cracking spree where they break into top-secret systems. The Computer Fraud and Abuse Act of 1986 was created because of Ian Murphy's crime of stealing information from military computers. A graduate student, Robert Morris, was convicted for unleashing the Morris Worm to over 6,000 vulnerable computers connected to the Internet. Based on concerns that the Morris Worm ordeal could be replicated, the Computer Emergency Response Team (CERT) was created to alert computer users of network security issues. 
In the 1990s, Internet became public and the security concern sincreased tremendously. Approximately950 million people use the internet today worldwide [3].On any day, there are approximately225majorincidencesofasecuritybreach [3]. These security breaches could also result in monetary losses of a large degree. Investment in proper security should be apriority for large organizations as well as common users.

\section{Internet architecture and Vulnerable security aspects}

Fear of security breaches on the Internet is causing organizations to use protected private networks or intranets [4]. The Internet Engineering Task Force (IETF) has introduced security mechanisms at various layers of the Internet Protocol Suite [4]. These security mechanisms allow for the logical protection of data units that are transferred across the network.

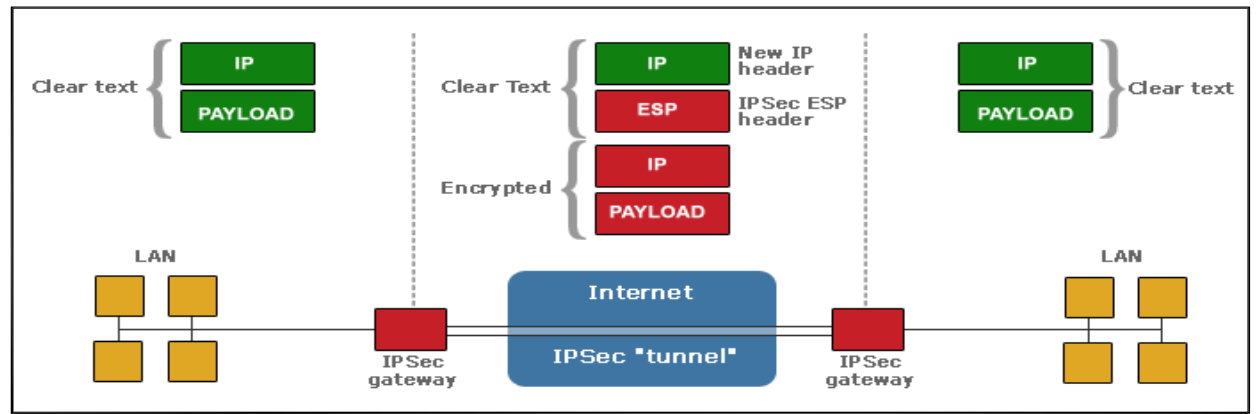

Figure2: IPsec contains a gateway and a tunnelinorder to secure communications. [17]

The security architecture of the internet protocol, known as IP Security, is a standardization of internet security. IP security, IP sec, covers the new generation of IP (IPv6) as well as the current version(IPv4). Although new techniques, such as IP sec, have been developed to overcome internet's best-known deficiencies, they seem to be insufficient [5]. Figure 2 show savisual representation of how IP sec is implemented to provide secure communications. IP Sec is a point-to-point protocol, one side encrypts, the other decrypts and both sides share key or keys. IP Sec can be used in two modes, namely transport mode and tunnel modes. The current version and new version of the Internet Protocol are analyzed to determine the security implications. Although security mayexist with in the protocol, certain attacks can not be guarded against. The se attacks are analyzed to determine other security mechanisms that may be necessary. $\mathbf{1}$.

\section{IPv4andIPv6Architectures}

IPv4 was design in 1980 to replace the NCP protocol on the ARPANET. The IPv4 displayed many limitations after two decades [6].TheIPv6 protocol was designed with IPv4's shortcomings in mind. IPv6 is not as upper set of the IPv4 protocol; instead it is a new design. The internet protocol's design is sovast and cannot be covered fully. The main parts of the architecture relating to security are discussed in detail.

\subsection{IPv4Architecture}

The protocol contains a couple aspects which caused problems with it suse. These problems do not all relate to security. They are mentioned to gain a comprehensive understanding of the internet protocol and its shortcomings. The causes of problems with the protocol are:

1. Address Space

2. Routing

3. Configuration

4. Security

5. Quality of Service

The IPv4 architecture has an address that is 32 bits wide[6]. This limits the maximum number of computers that can be connected to the internet. The 32 bit address provides for a maximum of two billions computers to be connected to the internet. The problem of exceeding that number was not foreseen when the protocol was created. The small address space of the IPv4 facilitates malicious code distribution[5]. Routing is a problem for this protocol because the routing tables are constantly increasing in size. The maximum the oretical size of the global routing tables was 2.1 million entries[6]. Methods have been a dopted to reduce the number of entries in the routing table. This is helpful for a short period of time, but drastic change needs to be made to address this problem. The TCP/IP- based networking of IPv4 requires that the user supplies some data in order to configure a network. Some of the information required is the IP address, routing gateway address, subnet mask, and DNS server. The simplicity of configuring the network is not evident in the IPv4 protocol. 
The user can request appropriate network configuration from a central server [6].This eases configuration hassles for the user but not the network's administrators.

The lack of embedded security with in the IPv4 protocol has led to the many attacks seen today. Mechanisms to secure IPv4 do exist ,but there are no requirements for the iruse [6]. IP sec is a specific mechanism used to secure the protocol. IP sec secures the packet payloads by means of cryptography. IP sec provides the services of confidentiality, integrity, and authentication [6]. This form of protection does not account for the skilled hacker who may be able to break the encryption method and obtain the key.

When internet was created, the quality of service (QoS) was standardized according to the information that was transferred across the network. The original transfer of information was mostly text-based. As the internet expanded and technology evolved, other forms of communication began to be transmitted across the internet. The quality of service for streaming video sand music are much different than the standard text. The protocol does not have the functionality of dynamic QoS that changes based on the type of data being communicated [6].

\subsection{IPv6Architecture}

When IPv6 was being developed, emphasis was placed on aspects of theIPv4 protocol that needed to be improved. The development efforts were placed in the following areas:

1. Routing and addressing

2. Multi-protocol architecture

3. Security architecture

4. Traffic control

The IPv6 protocol's address space was extended by supporting 128 bit addresses. With128 bit

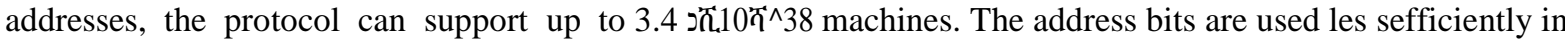
this protocol because its implifies addressing configuration. The IPv6 routing system is more efficient and enables smaller global routing tables. The host configuration is also simplified. Hosts can automatically configure themselves. This new design allow sease of configuration for the user as well as network administrator. The security architecture of theIPv6 protocol is of great interest. IP sec is embedded with in the IPv6 protocol. IP sec functionality is the same for IPv4 and IPv6. The only difference is that IPv6 canutilize the security mechanism along the entire route [6]. The quality of service problem is handled with IPv6. The internet protocol allows for special handling of certain packets with a higher quality of service. From a high-level view, the major benefits of IPv6 are its scalability and increased security. IPv6 also offers other interesting features that are beyond the scope of this paper.

It must be emphasized that after researching IPv6 and its security features, it is not necessarily more secure than IPv4.The approach to security is only slightly better, not a radical improvement.

\section{Attacks through the Current Internet Protocol IPv4}

There are four main computer security attributes. They were mentioned before in as lightly different form, but arere stated for convenience and emphasis. These security attributes are confidentiality, integrity, privacy, and availability. Confidentiality and integrity still hold to the same definition. Availability means the computer assets can be accessed by authorized people [8]. Privacy is the right to protect personal secrets [8]. Various attack methods relate to these four security attributes. Table1 shows the attack methods and solutions.

Table1: Attack Methods and Security Technology [8]

\begin{tabular}{|c|c|c|}
\hline $\begin{array}{c}\text { Computer } \\
\text { Security } \\
\text { attributes }\end{array}$ & Attack Methods & $\begin{array}{l}\text { Technology for } \\
\text { Intemet } \\
\text { Security }\end{array}$ \\
\hline Confidentiality & $\begin{array}{l}\text { Eavesdropping, } \\
\text { Ilacking, } \\
\text { Phishing, Dos } \\
\text { and IP Spoofing }\end{array}$ & $\begin{array}{l}\text { IDS, Firewall, } \\
\text { Cryptographic } \\
\text { Systems, IPSec } \\
\text { and SSL }\end{array}$ \\
\hline Integrity & $\begin{array}{l}\text { Viruses, Worms, } \\
\text { Trojans, } \\
\text { Eavesdropping, } \\
\text { DoS and IP } \\
\text { Spoofing. }\end{array}$ & $\begin{array}{c}\text { IDS, Firewall, } \\
\text { Anti-Malware } \\
\text { Software, IPSec } \\
\text { and SSL. }\end{array}$ \\
\hline Privacy & $\begin{array}{l}\text { Email bombing, } \\
\text { Spamming, } \\
\text { Hacking, DoS } \\
\text { and Cookies }\end{array}$ & $\begin{array}{c}\text { IDS, Firewall, } \\
\text { Anti-Malware } \\
\text { Software, IPSec } \\
\text { and SSL. }\end{array}$ \\
\hline Availability & $\begin{array}{c}\text { DoS, Email } \\
\text { bombing, } \\
\text { Spamming and } \\
\text { Systems Boot } \\
\text { Record Infectors }\end{array}$ & $\begin{array}{l}\text { IDS, Anti- } \\
\text { Malware } \\
\text { Software and } \\
\text { Firewall. }\end{array}$ \\
\hline
\end{tabular}


Common attack methods and the security technology will be briefly discussed. Not all of the methods in the table above are discussed. The current technology for dealing with attacks is understood in order to comprehend the current research developments in security hardware and software.

\subsection{CommonInternetAttackMethods}

Common internet attacks methods are broken down in to categories. Some attacks gain system knowledge or personal information, such as eavesdropping and phishing. Attacks can also interfere with the system's intended function, such as viruses, worms and trojans. The other form of attack is when the system's resources are consumes uselessly, the secan be caused by denial of service (DoS) attack. Other forms of network intrusions also exist, such as land attacks, smurf attacks, and teardrop attacks. These attacks are not as well known as DoS attacks, but they are used in some form or another evenif they aren't mentioned by name.

\subsubsection{Eavesdropping}

Interception of communications by an unauthorized party is called eaves dropping. Passive eaves dropping is when the persononly secretly listens to the networked messages. On the other hand, active eaves dropping is when the intruder listens and inserts something into the communication stream. This can lead to the messages being distorted. Sensitive information can be stolen this way [8].

\subsubsection{Viruses}

Viruses are self-replication programs that use files to infect and propagate [8]. Once a file is opened, the virus will activate with in the system.

\subsubsection{Worms}

A worm is similar to a virus because they both are self-replicating, but the worm does not require a file to allow it to propagate[8].There are two main types of worms, mass-mailing worms and network- aware worms. Mass mailing worms use email as a means to infect other computers. Network-aware worms are a major problem for the Internet. A network-aware worm selects a target and once the worm accesses the target host, it can infect it by means of a Trojan or otherwise.

\subsubsection{Trojans}

Trojans appear to be benign programs to the user, but will actually have some malicious purpose. Trojans usually carry some payload such as a virus [8].

\subsubsection{Phishing}

Phishing is an attempt to obtain confidential information from an individual, group, or organization [9]. Phisher strick users in to disclosing personal data, such a scredit card numbers, online banking credentials, and other sensitive information.

\subsubsection{IP Spoofing Attacks}

Spoofing means to have the address of the computer mirror the address of a trusted computer in order to gain access to other computers. The identity of the intruder is hidden by different means making detection and prevention difficult. With the current IP protocol technology, IP-spoofed packets cannot be eliminated [8].

\subsubsection{Denial of Service}

Denial of Service is an attack when the system receiving too many requests can not return communication with the requestors [9]. The system then consumes resources waiting for the handshake to complete. Eventually, the system cannot respond to any more requests rendering it without service.

\subsection{TechnologyforInternetSecurity}

Internet threats will continue to be a major issue in the global world as long as information is accessible and transferred across the Internet. Different defense and detection mechanisms were developed to deal with these attacks.

\subsubsection{Cryptographic systems}

Cryptography is a useful and widely used tool in security engineering today. It involved the use of codes and ciphers to transform information into unintelligible data. 


\subsubsection{Firewall}

A firewall is a typical border control mechan is morperimeter defense. The purpose of a firewall is to block traffic from the outside, but it could also beused to block traffic from the inside. A firewall is the front line defense mechanism against intruders. It is a system designed to prevent unauthorized access to or from a private network. Firewalls can be implemented in both hardware and software, or a combination of both [8].

\subsubsection{Intrusion Detection Systems}

An Intrusion Detection System (IDS) is an additional protection measure that helps ward off computer intrusions. IDS systems can be software and hardware devices used to detect an attack. IDS products are used to monitor connection in determining whether attacks are been launched. Some IDS systems just monitor and alert of an attack, whereas others try to block the attack.

\subsubsection{Anti-Malware Software and scanners}

Viruses, worms and Trojan horses are all examples of malicious software, or Malware for short. Special so-called anti-Malware toolsareused to detect them and cure an infected system.

\subsubsection{Secure Socket Layer (SSL)}

The Secure Socket Layer (SSL) is a suite of protocols that is a standard way to achieve a good level of security between a web browser and a website. SSL is designed to create a secure channel, or tunnel, between a web browser and the web server, so that any information exchanged is protected within the secured tunnel. SSL provides authentication of clients to server through the use of certificates. Clients present a certificate to the server to prove their identity.

\section{Security Issues of IP Protocol IPv6}

From a security point of view, IPv6 is a considerable advancement over the IPv4 internet protocol. Despite the IPv6's great security mechanisms, it still continues to be vulner able to threats. Some areas of the IPv6 protocol still pose a potential security issue. The new internet protocol does not protect against mis configured servers, poorly designed applications, or poorly protected sites. The possible security problems emerge due to the following [5]:

1. Header manipulation issues

2. Flooding issues

3. Mobility issues

Header manipulation issues a rise due to the IP sec's embedded functionality [7]. Extension headers deter some common sources of attacks because of header manipulation. The problem is that extension headers need to be processed by all stacks, and this can lead to a long chain of extension headers. The large number of extension headers can over whelm a certain node and is a form of attack if it is deliberate. Spoofing continues to be a security threat on IPv6protocol.

A type of attack called port scanning occurs when a whole section of a network is scanned to find potential targets with open services [5]. The address space of the IPv6 protocol is large but the protocol is still not in vulner able to this type of attack.

Mobility is a new feature that is incorporated in to the internet protocol IPv6.The feature requires special security measures. Network administrators need to be aware of these security needs when using IPv6's mobility feature.

\section{Security in different networks}

The businesses today use combinations of firewalls, encryption, and authentication mechanisms to create "intranets" that are connected to the internet but protected from it at the same time. Intranet is a private computer network that uses internet protocols. Intranets differ from "Extranets" in that the former are generally restricted to employees of the organization while extranets can generally be accessed by customers, suppliers, or other approved parties.

There does not necessarily have to be any access from the organization's internal network to the Internet itself. When such access is provided it is usually through a gateway with a firewall, along with user authentication, encryption of messages, and often makes use of virtual private networks (VPNs).

Although intranets can be setup quickly to share data in a controlled environment, that data is still a trisk unless there is tight security. The disadvantage of a closed intranet is that vital data might not get into the hand sof those who need it. Intranets have a place with in agencies. But for broader data sharing, it might be better to keep the networks open, with these safeguards:

1. Firewalls that detect and report intrusion attempts

2. Sophisticated virus checking at the firewall 
3. Enforcedrules for employee opening of e-mail attachments

4. Encryption for all connections and data transfers

5. Authentication by synchronized, timed passwords or security certificates

It was mentioned that if the intranet wanted access to the internet, virtual private networks are often used. Intranets that exist across multiple locations generally run over separate leased lines or a newer approach of VPN can be utilized. VPN is a private network that uses a public network (usually the Internet) to connect remote sites or users together. Instead of using a dedicated, real-world connection such as leased line, a VPN uses "virtual" connections routed through the Internet from the company's private network to there motesite or employee. Figure3is a graphical representation of an organization and VPN network.

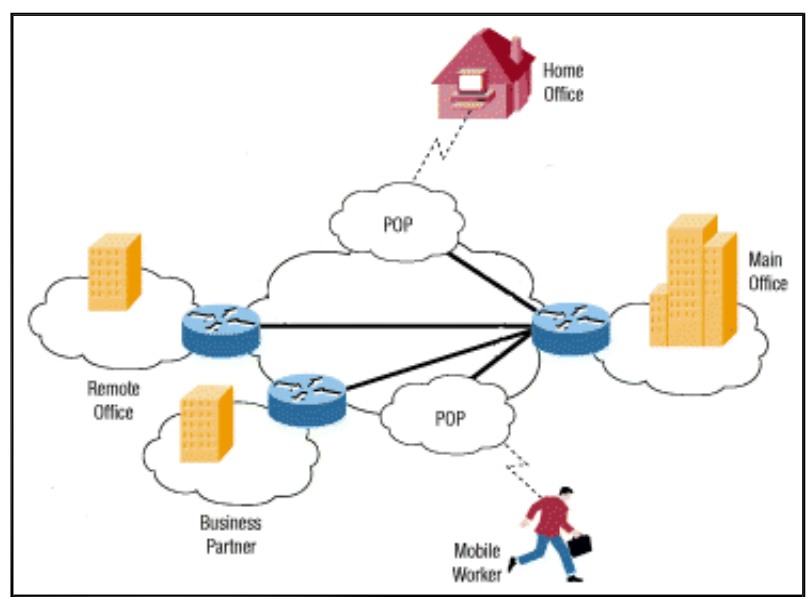

Figure3: A typical VPN might have a main LAN at the corporate headquarters of a company, other LANs at remote offices or facilities and in dividualusers connecting from out in the field.[14]

\section{Current developments in network Security}

The network security field is continuing down the same route. The same methodologies are being used with the addition of biometric identification. Biometrics provides a better method of authentication than passwords. This might greatly reduce the unauthorized access of secure systems. New technology such as the smart card is surfacing in research on network security. The software aspect of network security is very dynamic. Constantly new firewalls and encryption schemes are being implemented.

There search being performed assists in understanding current development and projecting the future developments of the field.

\section{Hardware Developments}

Hardware developments are not developing rapidly. Biometric systems and smartcards are the only new hardware technologies that are widely impacting security. The most obvious use of biometrics for network security is for secure work station logons for a workstation connected to a network. Each work station requires some software support for biometric identification of the user as well as, depending on the biometric being used, some hardware device. The cost of hardware devices is one thing that may lead to the widespread use of voice biometric security identification, especially among companies and organizations on alow budget. Hardware device such as computer mice with built in thumbprint readers would be the next step up. These devices would be more expensive to implement on several computers, as each machine would require its own hardware device. A biometric mouse, with the software to support it, is available from around $\$ 120$ in the U.S. The advantage of voice recognition software is that it can be centralized, thus reducing the cost of implementation permachine. At top of the range a centralized voice biometric package can cost up to $\$ 50,000$ butmaybeabletomanagethesecurelog- inof up to 5000 machines.

The mainuse of Biometric network security will be to replace the current password system. Main taining password security can be a major task for even a small organization. Passwords have to be changed every few months and people forget their password or lock themselves out of the system by incorrectly entering their password repeatedly. Very often people write their password down and keep it near their computer. This is of course completely undermines any effort at network security. Biometrics can replace this security identification method. The use of biometrici dentifications tops this problem and while it maybe expensive to setup at first, these devices save on administration and user assistance costs. 
Smart cards are usually acredit-card-sized digital electronic media. The card itself is designed to store encryption keys and other information used in authentication and other identification processes. The mainide a behind smart cards is to provide undeniable proof of a user's identity. Smart cards can be used for everything from logging in to the network to providing secure Web communications and secure e-mail transactions.

It may seem that smart cards are nothing more than are pository for storing passwords. Obviously, someone can easily steal a smart card from someone else Fortunately, there are safety features built in to smartcards to prevent someone from using a stolen card. Smart cards require anyone who is using them to enter a personal identification number (PIN) before they'll be granted any level of accessin to the system. The PIN is similar to the PIN used by ATM machines.

When a users inserts the smartcard in to the card reader, the smartcard prompts the user for a PIN. This PIN was assigned to the user by the administrator at the time the administrator issued the card to the user. Because the PIN is short and purely numeric, the user should have not roublere membering it and therefore would be unlikely to write the PIN down.

But the interesting thing is what happens when the user inputs the PIN. The PIN is verified from inside the smart card. Because the PIN is never transmitted across the network, there's absolutely no danger of it being intercepted. The main benefit, though, is that the PIN is useless without the smart card, and the smartcard isuse less without the PIN. There are other security issues of the smartcard. The smartcard is cost-effective but not a ssecure as the biometric identification devices.

\section{Software Developments}

The software aspect of network security is very vast. It includes firewalls, antivirus, vpn, intrusion detection, and much more. There search development of all security software is not feasible to study at this point. The goal is to obtain a view of where the security software is heading based on emphasis being placed now. The improvement of the standard security software still remains the same. When new viruses emerge, the antivirus is updated to be able to guard against those threats. This process is the same for firewalls and intrusion detection systems. Many research papers that have been skimmed were based on analyzing attack patterns in order to create smarter security software. As the security hardware transitions to biometrics, the software also needs to be able to use the information appropriately. Current research is being performed on security software using neural networks. The objective of there search is to use neural networks for the facial recognition software.

Many small and complex devices can be connected to the internet. Most of the current security algorithms are computational intensive and require substantial processing power. This power, however, is not available in small devices like sensors. Therefore, there is a need for designing light-weight security algorithms. Research in this area is currently being performed.

\section{Future trends in security}

What is going to drive the Internet security is the set of applications more than anything else. The future will possibly be that the security is similar to an immune system. The immune system fights off attacks and builds itself to fight tougher enemies. Similarly, the network security will be able to function as an immune system.

The trend towards biometrics could have taken place a while ago, but it seems that it isn't being actively pursued. Many security developments that are taking place are within the same set of security technology that is being used today with some minor adjustments.

\section{Conclusion}

Networks security is an important's field that is increasingly gaining attention as the Internet expands. The security threats and internet protocol were analyzed to determine the necessary security technology. The security technology is mostly software based, but many common hardware devices are used. The current development in network security is not very impressive. Originally it was assumed that with the importance of the network security field, new approaches to security, both hardware and software, would be actively researched. It was a surprise to see most of the development taking place in the same technologies being currently used. The embedded security of the new internet protocol IPv6 may provide many benefits to internet users. Although some security issues were observed, the IPv6 internet protocol seems to evade many of the current popular attacks. Combined use of IPv6 and security tools such as firewalls, intrusion detection, and authentication mechanisms will prove effective in guarding intellectual property for the near future. The network security field may have to evolve more rapidly to deal with the threats further in the future. 


\section{References}

[1]. Dowd, P.W.; Mc Henry, J.T.," Network security: it's time to takeitseriously,"Computer,vol.31,no.9,pp.24-

[2]. 28,Sep1998

[3]. Kartalopoulos,S.V.,"DifferentiatingDataSecurityandNetworkSecurity,"Communications, 2008.ICC'08.IEEEInternationalConference on,pp.1469-1473,19-23 May2008

[4]. Security Overview," www.redhat.com/docs/manuals/enterprise/RHEL-4- Manual/security-guide/ch-sgs-ov.html.

[5]. Molva,R.,InstitutEurecom,“InternetSecurityArchitecture,”inComputerNetworks\&ISDNSystems urnal,vol.31,pp.787-804,April1999

[6]. Sotillo, S.,EastCarolinaUniversity, "IPv6security issues,"August2006, www.infosecwriters.com/text_resources/pdf/IPv6_SSot illo.pdf.

[7]. Andress J., "IPv6:thenextinternetprotocol,"April 2005,www.usenix.com/publications/login/2005- 04/pdfs/andress0504.pdf

[8]. WarfieldM., "SecurityImplicationsofIPv6,"Internet SecuritySystems WhitePaper, documents.iss.net/whitepapers/IPv6.pdf

[9]. Adeyinka,O.,"InternetAttackMethodsandInternetSecurityTechnology,"Modeling\&Simulation,2008.AICMS08.SecondAsiaInternatio nalConferenceon,vol.,no.,pp.77-82,13-15May2008

[10]. Marin, G.A.," Network security basics, "Security \& Privacy,IEEE,vol.3,no.6,pp.68-72,Nov.-Dec.2005

[11]. "Internet History Timeline," www3.baylor.edu/ Sharon_P_Johnson/etg/inthistory.h tm.

[12]. Landwehr,C.E.;Goldschlag,D.M.,"SecurityissuesinnetworkswithInternetaccess,"ProceedingsoftheIEEE,vol.85,no.12,pp.2034-2051, Dec1997

[13]. Intranet."Wikipedia,TheFreeEncyclopedia.23Jun2008,10:43UTC.WikimediaFoundation,Inc.2Jul2008<http://en.wikipedia.org/w/in dex.php?title=Intranet\&ol did=221174244> .

[14]. Virtualprivatenetwork."Wikipedia,TheFreeEncyclopedia.30Jun2008,19:32UTC.WikimediaFoundation,Inc.2Jul2008<http://en.wiki pedia.org/w/index.php?title $=$ Virtual priv ate network\&oldid=222715612 > .

[15]. Tyson, J., "HowVirtualprivatenetworkswork,"http://www.howstuffworks.com/vpn.htm .

[16]. Al-Salqan,Y.Y.,"FuturetrendsinInternetsecurity,"DistributedComputingSystems, 1997.,ProceedingsoftheSixthIEEEComputerSociety WorkshoponFuture Trendsof,vol.,no.,pp.216-217,29-31Oct1997

[17]. Curtin,M.“IntroductiontoNetworkSecurity,"http://www.interhack.net/pubs/network-security.

[18]. Improving Security,"http://www.cert.org/tech_tips, 2006.

[19]. Serpanos,D.N.;Voyiatzis,A.G.,"Securenetworkdesign:Alayeredapproach,"AutonomousDecentralizedSystem,2002.The2ndInternatio nalWorkshopon,vol., no.,pp.95-100,6-7Nov.2002

[20]. Ohta,T.;Chikaraishi,T.,"Networksecuritymodel,"Networks, 1993.InternationalConferenceonInformationEngineering'93.'Communica tionsandNetworksfortheYear2000',ProceedingsofIEEESingaporeInternationalConferenceon,vol.2,no.pp.507-511vol.2,6-11Sep1993 